\title{
Expression and purification of FGF21 in Pichia pastoris and its effect on fibroblast-cell migration
}

\author{
YONGHUAN SONG ${ }^{1}$, JIAN DING $^{1}$, RILONG JIN $^{2}$, JINHEE JUNG $^{3}$, SHI LI $^{1}$, \\ JINGQUAN YANG ${ }^{1}$, ANYUAN WANG ${ }^{1}$ and ZHIJIE LI ${ }^{1}$ \\ ${ }^{1}$ Department of Hand and Plastic Surgery, The Second Affiliated Hospital of Wenzhou Medical University, \\ Wenzhou, Zhejiang 325027; ${ }^{2}$ Department of Orthopedic Surgery, The First Affiliated Hospital, \\ College of Medicine, Zhejiang University, Hangzhou, Zhejiang 310000, P.R. China; \\ ${ }^{3}$ Molecular Evolution Team, Department of Biotech R\&D, Amicogen Inc., Jinju, Yeongnam 660-852, South Korea
}

Received February 27, 2015; Accepted December 8, 2015

DOI: $10.3892 / \mathrm{mmr} .2016 .4942$

\begin{abstract}
Fibroblast growth factor (FGF)21 functions in the maintenance of glucose homeostasis and exerts protective effects on the liver, heat and kidneys. However, the roles of FGF21 in other tissue types are yet to be fully elucidated. The present study detected elevated expression levels of FGF21 in skin tissue. Furthermore, it was revealed that FGF21 expression in the skin was induced upon wounding. In addition, $\beta$-klotho expression was detected in the skin tissue. To examine the role of FGF21 in the wound healing process, recombinant human (h)FGF21 was expressed in a the yeast strain Pichia (P.) pastoris, a well-known system for recombinant protein production. Based on the sequence of hFGF21 and the optimal codon of P. pastoris, codon-optimized FGF21 open reading frame sequences were obtained using seven pairs of 55-59-nt primers with seven rounds of PCR. The recombinant FGF21 was purified and its function was examined in human fibroblast cells using a wound healing cell migration assay. Treatment with FGF21 promoted cell migration, which is an important step in wound healing. Furthermore, FGF21 treatment enhanced the activity of c-Jun N-terminal kinase, a key regulator in fibroblast-cell migration. In conclusion, FGF21 is induced after wounding and FGF21 expressed and purified from yeast markedly accelerates wound healing. The
\end{abstract}

Correspondence to: Professor Zhijie Li, Department of Hand and Plastic Surgery, The Second Affiliated Hospital of Wenzhou Medical University, 109 Xueyuan Road, Wenzhou, Zhejiang 325027, P.R. China

E-mail: 1zhjwh1@163.com

Abbreviations: FGF21, fibroblast growth factor 21; JNK, c-Jun $\mathrm{N}$-terminal kinase; SUMO, small ubiquitin-like modifier; MD, minimal dextrose; MM, minimal medium; Muts, methanol utilization slow; $\mathrm{Mut}^{+}$, methanol utilization plus; FGFR, fibroblast growth factor receptor; DEAE, diethylaminoethyl

Key words: fibroblast growth factor 21, yeast, expression, cell migration, c-Jun N-terminal kinase present study was the first to elucidate the function of FGF21 in skin tissues and provided a theoretical basis for the use of FGF21 in the treatment of skin wounds.

\section{Introduction}

The mammalian genome contains 23 fibroblast growth factors (FGFs) (1), which have essential roles in metabolism and development. FGFs have been identified to be involved in processes of embryogenesis, including gastrulation, somitogenesis, body plan formation and organogenesis, as well as in skin wound healing (2-7). Initially, 1, a member of the FGF family, was reported to be preferentially expressed in the liver (8). However, recent studies have identified that FGF21 is inducible by starvation or certain drugs, and have reported on its diverse functions in glucose homeostasis as well as hepatoand cardioprotection (9-11). FGF19, FGF21 and FGF23 belong to the FGF19 sub-family. Among them, FGF21 primarily activates FGF receptor (FGFR)1c, for which co-receptor $\beta$-klotho is required $(12,13)$.

Previous efforts to produce recombinant human FGF21 (rhFGF21) using an Escherichia (E.) coli system resulted in low expression of soluble protein, indicating that the majority of recombinant protein localized in inclusion bodies. Although the small ubiquitin-like modifier (SUMO) fusion system has been shown to facilitate the soluble expression and enhance the production of bioactive rhFGF21 (14), additional steps are required for the removal of tags from the protein expressed in vitro. At present, Pichia (P.) pastoris (yeast) is among the most successful eukaryotic protein expression systems. Compared with mammalian cells, culture of $P$. pastoris requires simple and cheap growth media and conditions, and $P$. pastoris is known to secrete proteins via signaling peptide-mediated secretion pathways. These features render the $P$. pastoris system suitable and efficient for recombinant protein expression (15).

In the present study rhFGF21 was expressed in P.pastoris cells and isolated from the culture media. Furthermore, examination of the expression of rhFGF21 in a variety of tissue types revealed elevated expression of FGF21 in skin compared with other tissue types, including that in the liver. 
Further experiments showed that FGF21 was induced by wounding and that exogenous treatment with FGF21 promoted cell migration, a key step of the wound healing process. The present study provided a novel method to express FGF21, which was not only more efficient than previous systems, but also provided a basis for FGF production in general. In addition, FGF21 accelerated fibroblast-cell migration, which suggested its applicability in the treatment of skin wounds.

\section{Materials and methods}

Creation of skin wounds on mice. Twelve male C57/BL6J mice, aged three months old and weighing 28-35 g, were obtained from the Laboratory Animal Centre of Wenzhou Medical University (Wenzhou, China), were divided into two equal groups. All mice were anesthetized via intraperitoneal injection of $3 \%$ sodium pentobarbital $(45 \mathrm{mg} / \mathrm{kg})$ and their dorsal areas were completely depilated using $\mathrm{Na}_{2} \mathrm{~S}(8.0 \%$; w/v) (both Sigma-Aldrich, St. Louis, MO, USA). Heart, liver, kidney and skin tissues were harvested from one group to analyze FGF21 tissue distribution; whereas skin wounds (length, $1 \mathrm{~cm}$; depth, $0.3 \mathrm{~cm}$ ) were created on the backs of the mice in the remaining group using a sharp laser blade (Swann-Morton, Sheffield, UK) to perforate the hypodermis. At 0 and $3 \mathrm{~h}$ following wound creation, $1 \mathrm{~cm}^{2}$ of the dorsal area surrounding the wound was collected in order to analyze the expression patterns of FGF21 and $\beta$-koltho. No mice were sacrificed before wounding. Following experimentation, mice were anesthetized using sodium pentobarbital and sacrificed via cervical dislocation.

Total RNA extraction, complementary DNA synthesis and polymerase chain reaction $(P C R)$. All frozen liver, heart, kidney and skin tissues were powdered using a mortar and pestle in liquid nitrogen and were homogenized for 30-45 sec in $1 \mathrm{ml}$ TRIzol reagent (Invitrogen; Thermo Fisher Scientific, Waltham, MA, USA) at increasing speed to the maximum setting on a IKA T-10 Basic Ultra Turrax homogenizer (IKA Werke GmbH \& Co. KG, Staufen, Germany). Following total RNA extraction with TRIzol reagent, RNA was quantified using a Nanodrop 2000 photometer (Thermo Fisher Scientific, Inc.). A total of $2 \mu \mathrm{g}$ total RNA was reverse-transcribed using a GoScript Reverse Transcription kit (Reverse Transcription System; Promega Corp., Madison, WI, USA) following the manufacturer's instructions. PCR was performed using a PCR Mastermix kit (Takara Biotechnology Co., Ltd., Dalian, China) on a T100 thermal cycler (Bio-Rad Laboratories, Inc., Hercules, CA, USA), as follows: $95^{\circ} \mathrm{C}$ for $5 \mathrm{~min}$, followed by 35 cycles of $94^{\circ} \mathrm{C}$ for $30 \mathrm{sec}$ and $58^{\circ} \mathrm{C}$ for $30 \mathrm{sec}, 72^{\circ} \mathrm{C}$ for $30 \mathrm{sec}$, elongation at $72^{\circ} \mathrm{C}$ for $5 \mathrm{~min}$ and holding at $10^{\circ} \mathrm{C}$. Gene expression levels were quantified as described previously (16). mRNA levels were normalized against those of GAPDH (cat. no. ab9482; 1:2500 dilution; Abcam, Cambridge, UK) using Image J software (National Institutes of Health, Bethesda, MD, USA) and the $2^{-\Delta \Delta C q}$ method (17). Gene-specific primer sequences were synthesized by Sangon Biotech Co., Ltd., (Shanghai, China), as follows: FGF21 forward, 5'-GAT GACGACCAAGACACTG-3' and reverse, 5'-CGGCCCTGT AAAGGCTCT-3'; $\beta$-klotho forward, 5'-ACGACCCGACGA GGGCTGTT-3' and reverse, 5'-GGAGGAGACCGTAAA
CTCGGGCTTA-3' and GAPDH forward, 5'-GCACAGTCA AGGCCGAGAAT-3' and reverse, 5'-GCCTTCTCCATG GTGGTGAA-3'. For the examination of PCR products size, a DL 2000 DNA ladder (Takara Biotechnology Co., Ltd.) was used in the present study.

Construction of recombinant expression plasmids. Based on the sequences of the mature human polypeptide of FGF21, which were obtained from NCBI, a FGF21 coding sequence was designed by replacing the non-preferred codon of $P$. pastoris with the corresponding preferred one (Table I) using Vector NTI Advance 10.0 software (Thermo Fisher Scientific, Inc.). Primers were synthesized by Sangon Biotech Co., Ltd., which also confirmed the correct insertion and reading frame of FGF21 by DNA sequencing. The sequences for the synthesis of the optimized-codon human FGF21 gene are stated in Table II. Pyrobest DNA polymerase $(0.05 \mathrm{U} / \mu \mathrm{l}$; Takara Biotechnology Co., Ltd.) was used for polymerase chain reaction (PCR) and codon-optimized FGF21 open reading frame sequences were obtained using seven pairs of 55-59-nt primers with seven rounds of PCR. The first round of PCR was performed using the P1/RP1 primer pair and P2/RP2 was used for the second round, with the previous round of PCR product used as template, and so forth. PCR thermal cycling was completed as follows: $94^{\circ} \mathrm{C}$ for $4 \mathrm{~min}$, followed by 32 cycles for $94^{\circ} \mathrm{C}$ for $30 \mathrm{sec}, 56^{\circ} \mathrm{C}$ for $30 \mathrm{sec}, 72^{\circ} \mathrm{C}$ at $30 \mathrm{sec}$, and extension ay $72^{\circ} \mathrm{C}$ for $5 \mathrm{~min}$. An XhoI restriction site was introduced during primer synthesis to allow for in-frame cloning into the a-factor secretion signal sequences containing expression vector pPIC9K (Invitrogen) in order to express the native N-terminus of FGF21. Furthermore, a nucleotide sequence encoding the KEX2 cleavage site was placed ahead of FGF21. At the C-terminus, a stop codon was introduced along with an EcoRI site. Synthesized FGF21 was cloned into $X h o I$ and EcoRI sites $(0.3 \mathrm{U} / \mu \mathrm{l})$ in the pPIC9K vector.

Yeast transfection, protein expression and purification. The plasmid pPIC9K-FGF21 DNA was linearized by $0.3 \mathrm{U} / \mu \mathrm{l}$ SalI and then electroporated into the P. pastoris strain SMD1168 (Invitrogen) at $1.5 \mathrm{KV}, 25 \mu \mathrm{F}$ and $200 \Omega$ using a Gene Pulser apparatus (Bio-Rad Laboratories, Inc.). After electroporation, $1 \mathrm{ml}$ of ice-cold $1 \mathrm{M}$ sorbitol was immediately added to the cells. The cells were incubated for $2 \mathrm{~h}$ at $30^{\circ} \mathrm{C}$ and transfected cells were selected on minimal dextrose (MD) plates $\left[1.34 \%\right.$ yeast nitrogen base medium (YNB), 4x10 $0^{-5} \%$ biotin, $1.5 \%$ agar (all Difco; BD Biosciences, Franklin Lakes, NJ, USA) and $2 \%$ dextrose (Sigma-Aldrich)] at $30^{\circ} \mathrm{C}$ for 2-3 days. The methyl utilization plus $\left(\mathrm{Mut}^{+}\right)$and methyl utilization slow $\left(\mathrm{Mut}^{\mathrm{s}}\right)$ phenotypes of the transfected cells were screened by replica-plating them onto minimal medium (MM) plates $\left(1.34 \%\right.$ YNB, $4 \times 10^{-5} \%$ biotin, $0.5 \%$ methanol (Sigma-Aldrich) as the primary carbon source and $1.5 \%$ agar) and MD plates to determine the methanol-utilizing phenotypes. Verification by PCR was performed using alcohol oxidase (AOX)1 universal primers (Sangon Biotech Co., Ltd.).

The $\mathrm{Mut}^{+}$and $\mathrm{Mut}^{\mathrm{s}}$ strains were subjected to shake-flask cultivation. Six positive colonies were selected and cultured in $100 \mathrm{ml} \mathrm{BMGYH}$ [2\% peptone, $1 \%$ yeast extract, $1.34 \%$ YNB, $100 \mathrm{mM}$ potassium phosphate (pH 6.0), 1\% glycerol, 
Table I. Unpreferred and preferred codons based on the codon bias of Pichia pastoris.

\begin{tabular}{lccccccccc}
\hline Amino acid & Leucine & Proline & Glutamic acid & Glutamine & Serine & Alanine & Glycine & Leucine & Threonine \\
\hline Unpreferred & CTG & CCG & GAG & CAG & AGC & GCC & GGC & CTG & ACG \\
Preferred & TTA & CCA & GAA & CAA & TCA & GCA & GGT & TTG & ACT \\
\hline
\end{tabular}

Table II. Primer sequences.

\begin{tabular}{ll} 
Primer & Sequence $\left(5^{\prime}-3^{\prime}\right)$ \\
\hline P1 & TGCCAACGCCCAGACGGGGCTCTCTATGGCAGTCTTCACTTCGATCCGGAAGCCTGTTC \\
P2 & GTGTGATTCAGATACTTGGCGTAAAAACCTCCCGTTTCTTATGCCAACGCCCAGACGGG \\
P3 & TCACCCGAATCTCTCTTGCAACTAAAAGCCTTGAAGCCTGGTGTGATTCAGATACTTG \\
P4 & GATTCGAGAAGATGGAACTGTTGGTGGAGCCGCAGACCAGTCACCCGAATCTCTCTTGC \\
P5 & CTGTATACAGATGACGCACAGCAAACGGAGGCACATCTCGAGATTCGAGAAGATGGAA \\
P6 & CCCACTACTTCAATTTGGGGGGCAGGTGAGGCAACGATACCTGTATACAGATGACGCAC \\
P7 & CCGCTCGAGAAAAGACATCCCATACCTGATAGCTCCCCACTACTTCAATTTGG \\
RP1 & ATACGTTGTATCCATCTTCCAAGAGAAGCTCACGAAATGAACAGGCTTCCGGATCG \\
RP2 & GCCAGGCAGGTGCAGTGGTAAACCATGCGCCTCACTCTGATATACGTTGTATCCATCTT \\
RP3 & GCAGGACCCCTAGGTGCTGGGTCCCGGTGTGGGCTCTTATTGCCAGGCAGGTGCAGTG \\
RP4 & CGGCAAAGCGGGAGGTAGGCCCGGTAATGGTAAAAATCTCGCAGGACCCCTAGGTGCTG \\
RP5 & TCCGACGTCGGGCGGCTGTGGAGCTAGGATTCCAGGGGGTTCCGGCAAAGCGGGAGGTA \\
RP6 & TGAGAGGGCCCAACCATCGACAGCGGATCACTAGATCCGACGTCGGGCGGCTGT \\
RP7 & GGAATTCCTTAGCTCGCGTATGACGGCGATCTACCTTGAGAGGGCCCAACCATCG
\end{tabular}

$0.004 \%$ histidine and $1.61 \mu \mathrm{M}$ biotin] in a 500-ml shaking flask until $\mathrm{OD}_{600}=5-6$ at $30^{\circ} \mathrm{C}$. Following centrifugation at $1,000 \mathrm{x} \mathrm{g}$ for $5 \mathrm{~min}$, the pellet was re-suspended to achieve an $\mathrm{OD}_{600}$ of 1.0 in $300 \mathrm{ml}$ BMMYH (BMGYH with $1 \%$ glycerol replaced with $0.8 \%$ methanol) to induce expression. Subsequently, cells were cultured for $72 \mathrm{~h}$ with addition of $0.8 \%$ methanol every $24 \mathrm{~h}$. Proteins were separated by $15 \%$ sodium dodecyl sulfate polyacrylamide gel electrophoresis (SDS-PAGE; Beyotime Institute of Biotechnology, Shanghai, China) and western blot analysis was performed using mouse anti-FGF21 monoclonal antibody (1:1,000; MAB25371; R\&D Systems, Inc., Minneapolis, MN, USA) (18).

Following centrifugation of the yeast cells, the supernatant $(300 \mathrm{ml})$ was subjected to sequential precipitation by slow addition of $142.8 \mathrm{~g}$ ammonium sulfate (Sigma-Aldrich) to a final concentration of $70 \%$ saturation over $2 \mathrm{~h}$. Following agitation overnight at $4{ }^{\circ} \mathrm{C}$, the suspension was centrifuged at $12,000 \mathrm{x}$ g for $20 \mathrm{~min}$ to harvest the protein precipitate, $100 \mathrm{ml}$ of which was re-suspended in $20 \mathrm{mM}$ phosphate buffer (PB; $\mathrm{pH} 8)$. The solution was dialyzed in $11 \mathrm{~PB}$ (20mM) overnight at $4^{\circ} \mathrm{C}$ using a dialysis bag (pore size, 3kD; Beijing Dingguo Changsheng Biotechnology Co., Ltd., Beijing, China) with three changes of PB. Subsequently, the solution was loaded, at a flow rate of $5 \mathrm{ml} / \mathrm{min}$, onto a $2.5 \times 15 \mathrm{~cm}$ diethylaminoethanol (DEAE)-Sepharose column (bed volume, $75 \mathrm{ml}$; GE Healthcare Life Sciences, Chalfont, UK), which had been equilibrated with five bed volumes of starting buffer containing $0.05 \mathrm{M} \mathrm{NaCl}$ and $20 \mathrm{mM} \mathrm{PB}$ (pH 8.0). Following washing with starting buffer, the bound proteins were eluted with a PB buffer gradient supplemented with $0.15,0.3,0.5$ and $1.0 \mathrm{M} \mathrm{NaCl}$. All fractions were collected and examined using 15\% SDS-PAGE, and the eluted fractions at $0.3 \mathrm{M} \mathrm{NaCl}$ containing FGF21 were further purified by a 2x80 cm Sephadex G-50 column (bed volume, $250 \mathrm{ml}$; GE Healthcare Life Sciences). The fraction containing FGF21 was concentrated to $5 \mathrm{ml}$ with polyethylene 2000. A total of $100 \mu \mathrm{l}$ of the concentrated filtrate was subjected to semi-preparative reverse-phase high-performance liquid chromatography (HPLC) using an Agilent Technologies 1200 series equipped with an Agilent 1260 Infinite Diode Array Detector (G4212B; ID, 2.1x100 mm; particle size, $2.5 \mu \mathrm{m}$; Agilent Technologies, Inc., NC, USA) on a $\mathrm{C} 18$ column pre-equilibrated with $0.1 \%$ trifluoroacetic acid (Merck Millipore, Darmstadt, Germany). Bound protein was eluted using a linear gradient of acetonitrile (10-90\%; Merck Millipore) in $0.1 \%$ TFA at a flow rate of $1.35 \mathrm{ml} / \mathrm{min}$ and monitored at $280 \mathrm{~nm}$ using a diode-array . The purity and integrity of FGF21 purified by reverse-phase HPLC was analyzed by determining the percentage of the major peak in the total area and electrospray ionization mass spectrometry (Bruker Reflex III MALDI-TOF MS, Bremen, Germany) with nitrogen at $337 \mathrm{~nm}$ (19). The fraction containing FGF21 at a purity of $>90 \%$ was subsequently lyophilized and the powder was stored at $-70^{\circ} \mathrm{C}$ for subsequent use.

Human foreskin fibroblast cell culture. Fibroblast culture was performed as previously described (20). Briefly, human foreskin samples $\left(2.1-3.6 \mathrm{~cm}^{2}\right)$ were collected 
from six individuals aged between 14 and 26 years at the Department of Dermatology at The Second Affiliated Hospital, Wenzhou Medical University between January and February 2014. The protocol of the present study was approved by the Institutional Ethical Committee of the Second Affiliated Hospital, Wenzhou Medical University (Wenzhou, China). Written informed consent was obtained from the patient. Following the removal of all fat, the tissue was cut into 3-mm/2-mm (length/width) strips and incubated in Dulbecco's modified Eagle's medium (DMEM; Hyclone, Logan, UT, USA) supplemented with $10 \%$ fetal bovine serum (FBS; Hyclone), $1 \%$ penicillin-streptomycin (Gibco; Thermo Fisher Scientific) and $0.05 \%$ dispase (Sigma-Aldrich) at $4^{\circ} \mathrm{C}$ overnight. Subsequently, the epidermis was removed and the dermis was harvested in $25-\mathrm{cm}^{2}$ flasks pre-treated with FBS, which were stored horizontally for $1 \mathrm{~h}$ and vertically for $2 \mathrm{~h}$ in a culture chamber containing $5 \% \mathrm{CO}_{2}$ at $37^{\circ} \mathrm{C}$. The tissues were cultured in DMEM, containing $5.5 \mathrm{mM}$ glucose, $10 \%$ FBS and $1 \%$ penicillin-streptomycin, and the medium was changed every three days. Cultured cells were digested and passaged with $0.25 \%$ trypsin (Gibco) once cell confluence reached $\sim 80 \%$. Cells at passage $4-5$ were used in the subsequent experiments.

Wound healing assay. The effects of FGF21 on the migratory capacity of fibroblasts was determined using the wound healing scratch assay, as previously described (20). Primary fibroblast cells were seeded onto 6-well plates at $80-90 \%$ confluence and incubated overnight in DMEM containing $0.5 \%$ FBS and $5 \mu \mathrm{g} / \mathrm{ml}$ mitomycin-C (Sigma-Aldrich). Linear scratch wounds were subsequently created in the confluent fibroblast monolayer using a sterile $200 \mu \mathrm{l}$ pipette tip (MFLab, Ningbo, China). The medium was immediately replaced with prewarmed $\left(37^{\circ} \mathrm{C}\right)$ fresh DMEM containing $0.5 \%$ FBS and $5 \mu \mathrm{g} / \mathrm{ml}$ mitomycin-C. Following $6 \mathrm{~h}$ culture, $100 \mathrm{ng} / \mathrm{ml} \mathrm{FGF} 21$ protein was added to the culture medium and gently shaken. At 0 and $24 \mathrm{~h}$ after wounding, images were captured using a microscope (IX70; Olympus, Tokyo, Japan) equipped with a CCD camera (CoolSNAP HQ; Nippon Roper, Chiba, Japan), which were controlled by MetaMorph 7.1 software (Molecular Devices, LLC, Sunnyvale, CA, USA). To quantify cell migration, 20 cells on the border of the wound area were randomly selected from each well and the migration distance was measured using ImageJ 14.8 software at the indicated time points (21).

Western blot analysis. Following the indicated treatments, cells were lysed in an ice-cold lysis solution (2 M thiourea, $7 \mathrm{M}$ urea, 2\% 3-[(3-cholamidopropyl)dimethylammonio]-1propanesulfonate, $40 \mathrm{mM}$ dithiothreitol, $40 \mathrm{mM}$ Tris base and $1 \%$ protease inhibitor; Sigma-Aldrich). Following centrifugation at $15,000 \mathrm{x} \mathrm{g}$ for $15 \mathrm{~min}$, the concentration of total protein in the supernatant was determined using the Bradfold protein assay (Bio-Rad Laboratories, Inc.). A total of $25 \mu \mathrm{g}$ protein was separated using $15 \%$ SDS-PAGE, followed by electrotransfer onto Immobilon-P transfer membranes (Millipore, Billerica, MA, USA). Subsequent to blocking in Tris-buffered saline containing $5 \%$ skimmed milk and $0.05 \%$ Tween-20 for $1 \mathrm{~h}$, membranes were blotted with the following primary rabbit monoclonal antibodies
A

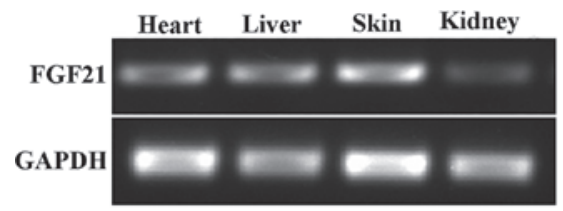

B

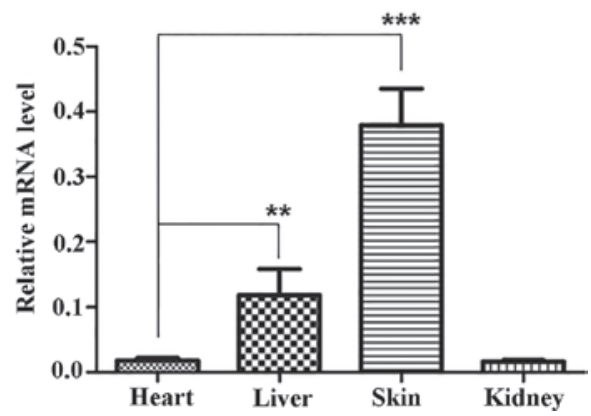

Figure 1. FGF21 expression patterns in a variety of mouse tissues. (A) Expression patterns of FGF21 were analyzed by reverse-transcription polymerase chain reaction analysis. (B) FGF21 levels in A were normalized against those of GAPDH. The experiments were repeated at least three times and values are expressed as the mean \pm standard deviation of three replicates. ${ }^{* *} \mathrm{P}<0.01 ;{ }^{* * *} \mathrm{P}<0.001$ vs. heart FGF21 levels.

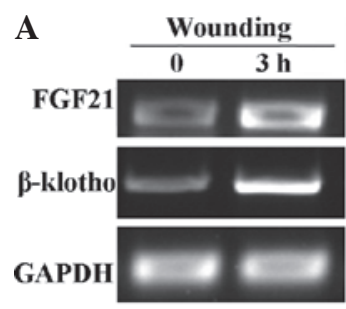

C
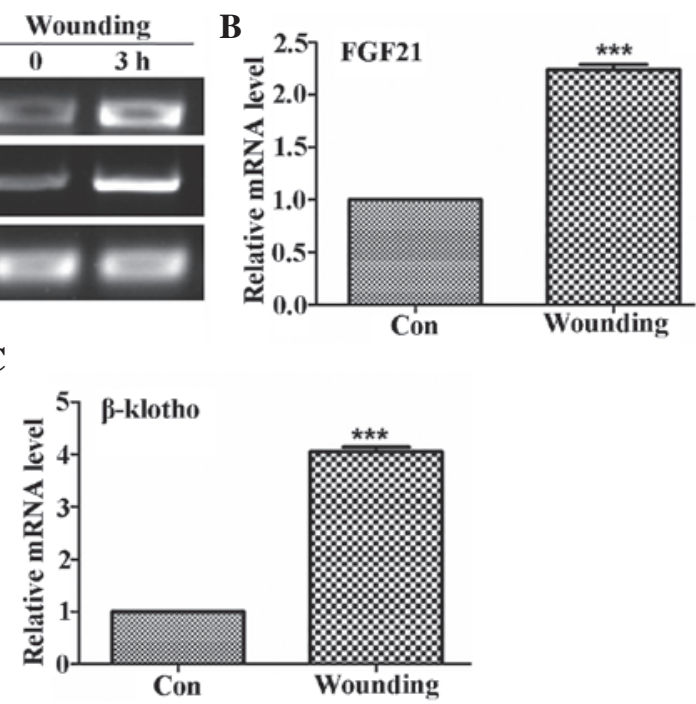

Figure 2. Expression patterns of FGF21 and $\beta$-klotho in skin following wounding. (A) Reverse-transcription polymerase chain reaction analysis was used to analyze the expression of FGF21 and $\beta$-klotho prior to and $3 \mathrm{~h}$ after wounding. Expression levels of (B) FGF21 and (C) $\beta$-klotho were normalized against those of GAPDH. The experiments were repeated at least three times and values are expressed as the mean \pm standard error of three replicates. ${ }^{* * * *} \mathrm{P}<0.001$ vs. Con. Con, control.

at $4^{\circ} \mathrm{C}$ overnight: Anti-phospho (p)-stress-activated protein kinase/c-Jun N-terminal kinase (JNK) $\left(\mathrm{Thr}^{183} / \mathrm{Ty}^{185}\right.$ ) (cat. no. 4668; 1:1,000 dilution; Cell Signaling Technology, Inc., Danvers, MA, USA), anti-JNK (cat. no. ab179461; 1:1,2000) and anti-FGF21 (cat. no. ab171941; 1:2,000 dilution; both Abcam). Following three washes with TBST, the membranes were incubated with horseradish peroxidase-conjugated goat anti-rabbit secondary antibody (cat. no. 7074; 1:2,000 dilution; Cell Signaling Technology, Inc.) for $1 \mathrm{~h}$ at room temperature. Antigen-antibody complexes were then visualized using an enhanced chemiluminescence kit (GE Healthcare Life Sciences). Images of the western 
blot and X-ray film were captured using an ImageQuant LAS 4000 Mini (GE Healthcare Life Sciences) and an Epson Perfection V700 photo scanner (Epson Corporation, Nagano, Japan). Protein levels were normalized against those of total total c-Jun N-terminal kinase (t-JNK) using Image J software. A protein molecular weight marker (Takara Biotechnology Co., Ltd.) was used to analyze protein size.

Statistical analysis. Statistical analysis was performed using two-way analysis of variance and Bonferroni post-hoc analysis on Prism 5 software (GraphPad, Inc., La Jolla, CA, USA). Comparison between two groups was performed using an unpaired Student's t-test. Values are expressed as the mean \pm standard error. $\mathrm{P}<0.05$ was considered to indicate a statistically significant difference between values.

\section{Results}

FGF21 is highly expressed in skin tissues and is upregulated following wounding. Initially, FGF21 has been reported to be preferentially expressed in the liver (8). To determine FGF21 expression patterns in other tissue types, the present study subjected liver, heart, kidney and skin samples to RT-PCR analysis of FGF21. The results showed that FGF21 is highly expressed in the liver; however, an even higher expression $(\sim 3$-fold) was detected in the skin (Fig. 1). Historical identification of the FGF family via the analysis of numerous tissues demonstrated that basic fibroblast growth factor (bFGF) was a major member of the fibroblast growth factor in the brain, fibroblasts and other tissues (22-24). Given that skin wound healing is a process regulated by various proteins and that bFGF is a member of the FGF gene family, which is widely known for its implication in wound healing $(7,21)$, the present study investigated the role of FGF21 in wound healing. Expression patterns of FGF21 and FGFR co-receptor $\beta$-klotho in the skin of mice were analyzed prior to and $3 \mathrm{~h}$ following wounding. As shown in Fig. 2, FGF21 expression in the skin of mice was $\sim 2$.2-fold increased following wounding, while $\beta$-klotho was induced by $\sim 4$-fold after wounding. These results indicated that FGF21 was highly expressed in the skin and induced by skin wounding.

Construction of expression vector. Previous studies have reported that expression of FGF21 in E. coli is challenging and inefficient $(25,26)$. To establish an alternative eukaryotic system for FGF expression with higher efficiency, a yeast system was utilized. Compared to bacteria, yeast is an eukaryotic organism with higher genetic similarity to humans; furthermore, yeast cells secrete proteins into the culture medium, allowing for soluble proteins to be harvested without the necessity of cell lysis. Translationally preferred codons are selected for accurate and efficient translation in bacteria, yeast, worm, fly, and mammals (27); therefore, the present study optimized the codon usage of the wild-type FGF21 gene based on codon bias of P.pastoris without changing the amino acid composition (Table I) and designed seven pairs of primers (Table II) to synthesize FGF21 open reading frame. The full length of FGF21 was obtained by employing seven PCR cycles (Fig. 3) and was cloned into the $X h o \mathrm{I} / E c o$ RI sites of the pPIC9K expression vector to yield

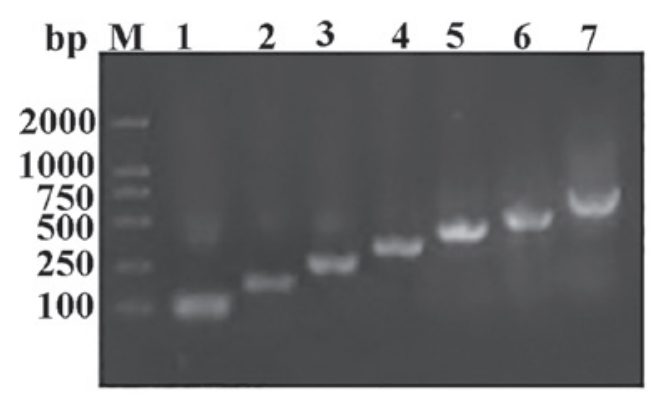

Figure 3. Gene synthesis of FGF21. PCR products of the gene FGF21. Lanes: M, 2000 DNA marker; 1, PCR reaction with primer pair P1/RP1; 2, PCR reaction with primer pair $\mathrm{P} 2 / \mathrm{RP} 2$ using the $\mathrm{PCR}$ product from the previous cycle as a template; 3-7, PCR reactions with primer pairs P3/RP3-P7/RP7, respectively, using the PCR product from the previous round as a template. $\mathrm{PCR}$, polymerase chain reaction.

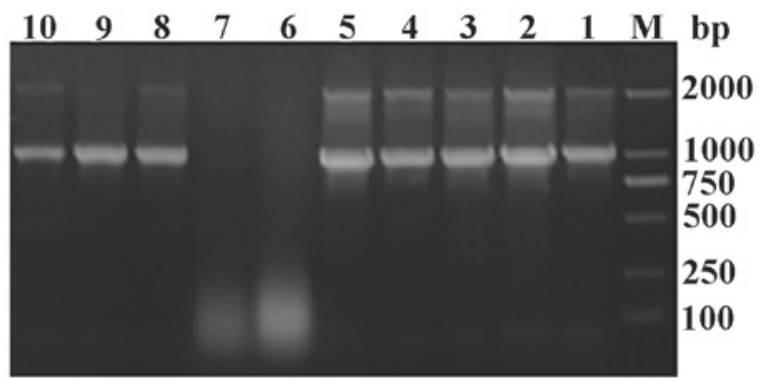

Figure 4. Polymerase chain reaction analysis confirmed that the FGF21 fragment was successfully integrated into SMD1168 colonies. Lanes 1-10 are colonies selected from a minimal dextrose plate. Yeast cells transfected with empty vector was used as a negative control. M, marker.

recombinant FGF21. Correct insertion and reading frame of FGF21 were confirmed by DNA sequencing.

Phenotype screening. After 2-3 days following transfection, the $\mathrm{Mut}^{+}$phenotypes grew normally on MM as well as on MD plates. Although Mut ${ }^{\mathrm{s}}$ phenotypes grew as well as $\mathrm{Mut}^{+}$on MD plates, the colony sizes of $\mathrm{Mut}^{\mathrm{s}}$ phenotype cells were smaller on MM plates, as compared with the MD plates at the same time point (data not shown). The Mut ${ }^{+}$cells expressed the AOX1 gene, which encodes alcohol oxidase, enabling recombinants to rapidly grow with methanol as the sole carbon source. However, due to a recombination at the AOX1 locus to result in loss of the AOX1 gene in the Mut ${ }^{\mathrm{s}}$ phenotype, the cells' ability to metabolize methanol and consequently their growth was reduced. The genomic DNA of the transformants was extracted and used as the template for PCR analysis. There were two fragments of $1.0-\mathrm{kb}$ product (containing the insert gene sequence of the 546- and 492-bp from the vector) and a $2.2-\mathrm{kb}$ product (AOX1 gene) which was amplified with the AOX1 universal primers for positive yeast transformants, whereas the negative recombinants exhibited only one fragment of $2.2 \mathrm{~kb}$, demonstrating that the recombinant expression vector had been successfully transfected into the yeast genome, while no inserted fragment was detected for negative yeast recombinants (Fig. 4).

Expression and purification of FGF21 in P. pastoris. The $\mathrm{Mut}^{+}(\mathrm{SMD} 1168)$ strains transfected with the FGF21 
A

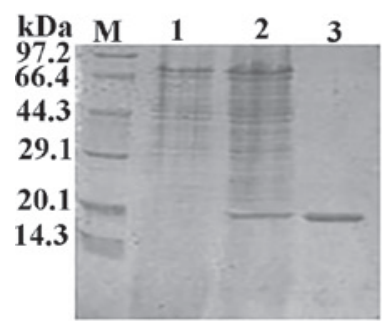

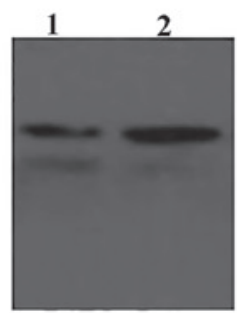

C

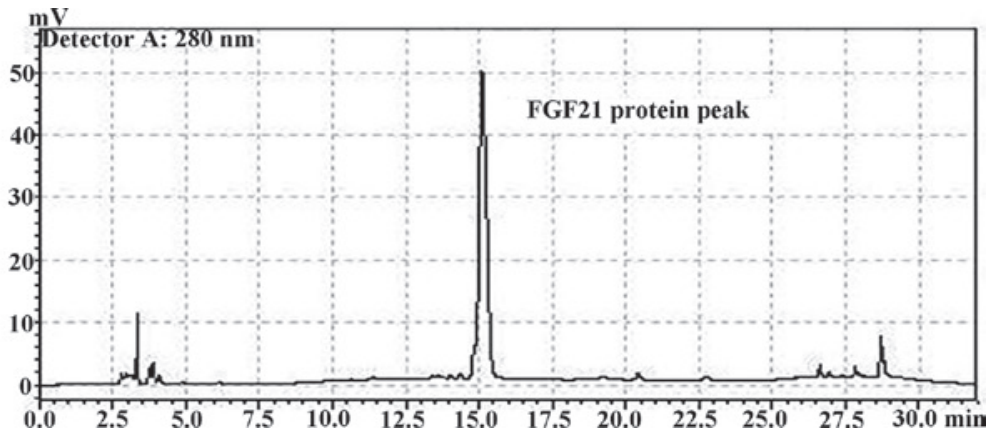

Figure 5. Protein expression and purification of FGF21. (A) SDS-PAGE analysis of supernatant of engineered P. pastoris cells and purified FGF21 protein. Lanes: 1, marker; 2, supernatant without transfection with the FGF21 overexpression vector; 3, supernatant after transfection; 4, purified protein. (B) Purified FGF21 protein was analyzed by western blotting using an Epson Perfection V700 photo scanner. Lanes: 1, protein in the supernatant after transfection; 2 , purified protein. (C) The purity of recombinant FGF21 was analyzed by high-performance liquid chromatography. FGF, fibroblast growth factor.
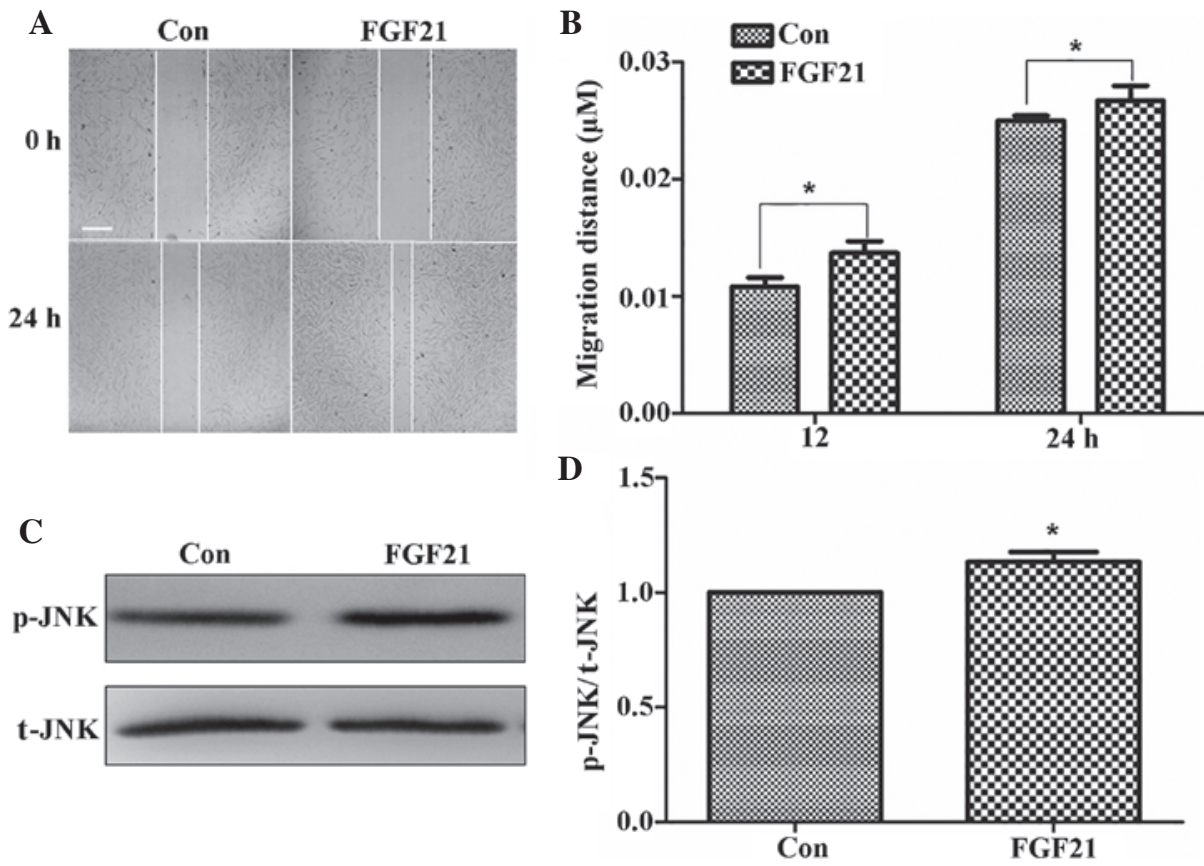

Figure 6. Effects of exogenous FGF21 treatment on the activation of cell migration and JNK phosphorylation levels. (A) Wound healing assay was performed to analyze the effects of FGF21 (100 ng/ml) in human fibroblasts under low-glucose $(5.5 \mathrm{mM})$ conditions. Scale bar, $0.05 \mu \mathrm{m}$. Magnification, $40 \mathrm{x}$. (B) The cell migration distance was quantified from A. (C and D) Following $6 \mathrm{~h}$ culture, $100 \mathrm{ng} / \mathrm{ml} \mathrm{FGF21} \mathrm{was} \mathrm{added} \mathrm{to} \mathrm{the} \mathrm{culture} \mathrm{medium} \mathrm{and} \mathrm{gently} \mathrm{shaken.} \mathrm{Phosphorylation}$ levels of JNK were analyzed after $30 \mathrm{~min}$ of FGF21 stimulation. All experiments were performed after incubation with $5 \mu \mathrm{g} / \mathrm{ml} \mathrm{mitomycin-C,} \mathrm{an} \mathrm{inhibitor} \mathrm{of}$ cell proliferation, for one day. Images were captured using an ImageQuant LAS 4000 . Values are expressed as the mean \pm standard error $(n=10$ for $B$ and $n=3$ for $\mathrm{D}$ ). ${ }^{\mathrm{P}} \mathrm{P}<0.05$ vs. control. p-JNK, phosphorylated c-Jun N-terminal kinase; t-JNK, total c-Jun N-terminal kinase; FGF, fibroblast growth factor; Con, control.

expression vector were incubated in flasks with agitation, and their FGF21 production was assessed. After $96 \mathrm{~h}$ of induction with $0.8 \%$ methanol, 11 cell culture supernatant containing FGF21 was collected and purified by ammonium sulfate precipitation, Sephadex G-50 gel filtration and DEAE-Sepharose ion-exchange chromatography. The putative FGF21 protein was analyzed by $15 \%$ SDS-PAGE, revealing a $\sim 20-\mathrm{kDa}$ product (Fig. 5A). Purified recombinant FGF21 was further confirmed by western blot analysis using an FGF21-specific antibody (Fig. 5B). Analytical HPLC was further employed to reveal that the FGF21 protein was obtained with $>90 \%$ purity (Fig. 5C). The yield of the recombinant protein $(\sim 15 \mathrm{mg} / \mathrm{l})$ was relatively low in $P$. pastoris, as compared with that of $E$. coli $(213 \pm 17 \mathrm{mg} / \mathrm{l})(28)$. These results demonstrated that FGF21 was successfully expressed in $P$. pastoris.
FGF 21 promotes fibroblast migration. The present study further investigated whether the produced and purified FGF21 exerted any effects on cell migration, which may be beneficial in wound healing. As FGF21 was highly expressed in the skin of mice and induced after wounding, its function in wound healing was further examined. The wound healing process comprises multiple steps, including proliferation and migration of fibroblasts, which are regulated by various proteins, including FGFs. To test effects of FGF21 on the cell migration process, human primary foreskin fibroblast cells were grown in low-glucose DMEM containing $5 \mu \mathrm{g} / \mathrm{ml}$ mitomycin-C to prevent cell proliferation for one day, following which the cell monolayers were wounded and incubated with $100 \mathrm{ng} / \mathrm{ml} \mathrm{FGF21.} \mathrm{As} \mathrm{shown} \mathrm{in}$ Fig. 6A and B, the cells treated with FGF21 displayed enhanced cell migration at $24 \mathrm{~h}$ following wound generation. 
FGF21 activates the JNK pathway in fibroblasts. JNK activation is associated with its phosphorylation and has an important role during cell migration during wound healing (20). Therefore, JNK phosphorylation in fibroblasts was examined following FGF21 treatment. FGF21 treatment activated p-JNK compared to that in the control group (Fig. 6C and D). FGF21 increased JNK activation, which may potentially represent the underlying mechanism by which it accelerates fibroblast-cell migration.

\section{Discussion}

Skin wound healing is a complex process that requires the actions of keratinocytes, fibroblasts, endothelial cells, macrophages and platelets. These cells undergo multiple steps, including proliferation and migration, to rebuild the skin (29). In this process, fibroblasts cause wound contraction, and fibroblast-cell migration is considered to be a fundamental step towards wound healing. Cell migration is a multi-step cyclic process, including extension of a protrusion, stable attachment to a site proximal to the leading edge of the protrusion, forward movement of the cell body, release of adhesions and retraction at the cell rear (30). bFGF is another member of the FGF family, whose efficacy in promoting fibroblast-cell migration has been previously described (7). The functions of FGF21 in glucose homeostasis as well as in hepato- and cardioprotection have been reported (9-11). While it has been reported that FGF21 is highly expressed in the liver (8), the present study revealed that its expression was even higher in the injured skin of mice. Following wounding, the expression of FGF21 and $\beta$-klotho, co-receptor of FGF21 necessary for activation of FGF signaling, was enhanced in the skin of mice, suggesting that FGF21 is involved in the wound healing process. Furthermore, recombinant FGF21 protein was identified to enhance the migration of human fibroblast cells, therefore exerting a similar function to that of bFGF (7). bFGF was shown to accelerate fibroblast migration via activation of the phosphoinositide-3 kinase/RacI/JNK pathway (7). In the present study, FGF21 application activated JNK phosphorylation in fibroblasts, which may be the underlying mechanism of its enhancing effects on cell migration. These findings led to the hypothesis that FGF21 promotes wound repair, and that the underlying mechanism include the activation of the JNK signaling pathway.

$E$. coli, the most widely used system for heterologous protein expression, has been previously used for FGF21 production $(14,25)$. This type of engineering uses polyethylene glycol or SUMO protein tags to increase the production and stability of soluble recombinant protein; however, this technique requires additional steps to remove the tags. In the present study, $P$. pastoris was used to express recombinant FGF21, which offers a simple technique for obtaining soluble proteins due to their secretion into the culture media. In the present study, only a small amount of recombinant FGF21 protein was isolated from the media, implying that FGF21 expression in this yeast system may still be low.

Of note, the FGF21 produced in the present study was demonstrated to promote wound healing in vitro. Furthermore, mechanistic analysis showed that recombinant FGF21 triggered the activation of JNK, a downstream regulator of FGFR. These findings suggested that FGF21 expressed by $P$. pastoris was biologically active.

Methods provided by previous studies may be utilized for designing strains for the production of specific proteins, including transcription and translation factors (31) or chaperones (32). Modification of yeast strains will enhance their capacity for protein production. Recently, a study on Saccharomyces cerevisiae indicated that the choice of auxotrophic marker was shown to be crucial for developing a yeast expression system with stable heterologous protein production (33). The present study was a first attempt to produce FGF21 using a yeast strain, and further engineering of yeast strains as well as additional optimizations will provide a platform for the efficient expression of proteins of interest, such as FGF21.

In conclusion, the present study demonstrated that FGF21 expression in skin tissue was higher compared to that in other tissues. Furthermore, FGF21 expression was identified to be induced following skin wounding. Exogenous treatment of fibroblasts with FGF21 produced by an engineered $P$. pastoris yeast strain promoted cell migration and activated JNK phosphorylation, a key regulator of wound healing. The present study therefore provided a novel eukaryotic system for the expression of FGFs and indicated that FGF21 may aid in accelerating skin wound healing.

\section{Acknowledgements}

The present study was supported by the Research Development Fund of Wenzhou Medical University (grant no. QTJ14029).

\section{References}

1. Mohammadi M, Olsen SK and Ibrahimi OA: Structural basis for fibroblast growth factor receptor activation. Cytokine Growth Factor Rev 16: 107-137, 2005.

2. Feldman B, Poueymirou W, Papaioannou VE, DeChiara TM and Goldfarb M: Requirement of FGF-4 for postimplantation mouse development. Science 267: 246-249, 1995.

3. Dubrulle J and Pourquié O: Fgf8 mRNA decay establishes a gradient that couples axial elongation to patterning in the vertebrate embryo. Nature 427: 419-422, 2004.

4. Sun X, Meyers EN, Lewandoski M and Martin GR: Targeted disruption of Fgf8 causes failure of cell migration in the gastrulating mouse embryo. Genes Dev 13: 1834-1846, 1999.

5. Martin GR: The roles of FGFs in the early development of vertebrate limbs. Genes Dev 12: 1571-1586, 1998.

6. Goldfarb M: Functions of fibroblast growth factors in vertebrate development. Cytokine Growth Factor Rev 7: 311-325, 1996.

7. Kanazawa S, Fujiwara T, Matsuzaki S, Shingaki K, Taniguchi M, Miyata S, Tohyama M, Sakai Y, Yano K, Hosokawa K and Kubo T: BFGF regulates PI3-kinase-Rac1-JNK pathway and promotes fibroblast migration in wound healing. PLoS One 5: e12228, 2010.

8. Nishimura T, Nakatake $\mathrm{Y}$, Konishi $\mathrm{M}$ and Itoh $\mathrm{N}$ : Identification of a novel FGF, FGF-21, preferentially expressed in the liver. Biochim Biophys Acta 1492: 203-206, 2000.

9. Liang Q, Zhong L, Zhang J, Wang Y, Bornstein SR, Triggle CR, Ding H, Lam KS and Xu A: FGF21 maintains glucose homeostasis by mediating the cross talk between liver and brain during prolonged fasting. Diabetes 63: 4064-4075, 2014.

10. Lin Z, Tian H, Lam KS, Lin S, Hoo RC, Konishi M, Itoh N, Wang Y, Bornstein SR, Xu A and Li X: Adiponectin mediates the metabolic effects of FGF21 on glucose homeostasis and insulin sensitivity in mice. Cell Metab 17: 779-789, 2013.

11. Lin Z, Wu F, Lin S, Pan X, Jin L, Lu T, Shi L, Wang Y, Xu A and $\mathrm{Li} \mathrm{X}$ : Adiponectin protects against acetaminophen-induced mitochondrial dysfunction and acute liver injury by promoting autophagy in mice. J Hepatol 61: 825-831, 2014. 
12. Yie J, Wang W, Deng L, Tam LT, Stevens J, Chen MM, Li Y, Xu J, Lindberg R, Hecht R, et al: Understanding the physical interactions in the FGF21/FGFR/ $\beta$-Klotho complex: Structural requirements and implications in FGF21 signaling. Chem Biol Drug Des 79: 398-410, 2012.

13. Belov AA and Mohammadi M: Molecular mechanisms of fibroblast growth factor signaling in physiology and pathology. Cold Spring Harb Perspect Biol 5: a015958, 2013.

14. Wang H, Xiao Y, Fu L, Zhao H, Zhang Y, Wan X, Qin Y, Huang $\mathrm{Y}, \mathrm{Gao} \mathrm{H}$ and $\mathrm{Li} \mathrm{X}$ : High-level expression and purification of soluble recombinant FGF21 protein by SUMO fusion in Escherichia coli. BMC Biotechnol 10: 14, 2010.

15. Daly R and Hearn MT: Expression of heterologous proteins in Pichia pastoris: A useful experimental tool in protein engineering and production. J Mol Recognit 18: 119-138, 2005.

16. Zittermann SI and Issekutz AC: Basic fibroblast growth factor (bFGF, FGF-2) potentiates leukocyte recruitment to inflammation by enhancing endothelial adhesion molecule expression. Am J Pathol 168: 835-846, 2006.

17. Livak KJ and Schmittgen TD: Analysis of relative gene expression data using real-time quantitative PCR and the $2^{-\Delta \Delta C t}$ method. Methods 25: 402-408, 2001.

18. Jin F, Xu X, Zhang W and Gu D: Expression and characterization of a housefly cecropin gene in the methylotrophic yeast, Pichia pastoris. Protein Expr Purif 49: 39-46, 2006.

19. Jin FL, Xu XX, Yu XQ and Ren SX: High-level expression of active recombinant ubiquitin carboxyl-terminal hydrolase of Drosophila melanogaster in Pichia pastoris. Protein Expr Purif 65: 115-121, 2009.

20. Xuan YH, Huang BB, Tian HS, Chi LS, Duan YM, Wang X, Zhu ZX, Cai WH, Zhu YT, Wei TM, et al: High-glucose inhibits human fibroblast cell migration in wound healing via repression of bFGF-regulating JNK phosphorylation. PLoS One 9: e108182, 2014.

21. Iyer VR, Eisen MB, Ross DT, Schuler G, Moore T, Lee JC, Trent JM, Staudt LM, Hudson J Jr, Boguski MS, et al: The transcriptional program in the response of human fibroblasts to serum. Science 283: 83-87, 1999.

22. Burgess WH and Maciag T: The heparin-binding (fibroblast) growth factor family of proteins. Annu Rev Biochemistry 58 575-602, 1989.
23. Courty J, Dauchel MC, Caruelle D, Perderiset M and Barritault D: Mitogenic properties of a new endothelial cell growth factor related to pleiotrophin. Biochem Biophys Res Commun 180: 145-151, 1991.

24. Fernig DG and Gallagher JT: Fibroblast growth factors and their receptors: An information network controlling tissue growth, morphogenesis and repair. Prog Growth Factor Res 5: 353-377, 1994.

25. Huang Z, Wang H, Lu M, Sun C, Wu X, Tan Y, Ye C, Zhu G, Wang $X$, Cai L and Li X: A better anti-diabetic recombinant human fibroblast growth factor 21 (rhFGF21) modified with polyethylene glycol. PLoS One 6: e20669, 2011.

26. Kharitonenkov A, Shiyanova TL, Koester A, Ford AM, Micanovic R, Galbreath EJ, Sandusky GE, Hammond LJ, Moyers JS, Owens RA, et al: FGF-21 as a novel metabolic regulator. J Clin Invest 115: $1627-1635,2005$.

27. Gu W, Zhou T and Wilke CO: A universal trend of reduced mRNA stability near the translation-initiation site in prokaryotes and eukaryotes. PLoS Comput Biol 6: e1000664-e1000664, 2010.

28. Zhang M, Jiang X, Su Z, Lin J, Xiang Q, Yang Z, Huang Y and Li X: Large-scale expression, purification, and glucose uptake activity of recombinant human FGF21 in Escherichia coli. Appl Microbiol Biotechnol 93: 613-621, 2012.

29. Martin P: Wound healing-aiming for perfect skin regeneration. Science 276: 75-81, 1997.

30. Cao C, Sun Y, Healey S, Bi Z, Hu G, Wan S, Kouttab N, Chu W and Wan Y: EGFR-mediated expression of aquaporin-3 is involved in human skin fibroblast migration. Biochem J 400: 225-234, 2006.

31. Dever TE: Using GCN4 as a reporter of eIF2 alpha phosphorylation and translational regulation in yeast. Methods 11: 403-417, 1997.

32. Payne T, Finnis C, Evans LR, Mead DJ, Avery SV, Archer DB and Sleep D: Modulation of chaperone gene expression in mutagenized Saccharomyces cerevisiae strains developed for recombinant human albumin production results in increased production of multiple heterologous proteins. Appl Environ Microbiol 74: 7759-7766, 2008.

33. Kazemi Seresht A, Nørgaard P, Palmqvist EA, Andersen AS and Olsson L: Modulating heterologous protein production in yeast: The applicability of truncated auxotrophic markers. Appl Microbiol Biotechnol 97: 3939-3948, 2013. 